\title{
Upper-Bound Performance of a Wide-Band Adaptive Modem
}

\author{
C. H. Wong and Lajos Hanzo, Senior Member, IEEE
}

\begin{abstract}
Adaptive modulation is applied in conjunction with a decision-feedback equalizer (DFE) in order to mitigate the effects of the slowly varying wide-band multipath Rayleigh fading channel in a noise-limited environment. An upper-bound mean bit-error rate and bits per symbol performance is introduced for this scheme by utilizing the pseudo signal-to-noise ratio at the output of the DFE in order to switch the modulation schemes on a burst-by-burst basis.
\end{abstract}

Index Terms-AQAM, QAM, quadrature amplitude modulation, wide-band adaptive modulation.

\section{INTRODUCTION}

A DAPTIVE quadrature amplitude modulation (AQAM) employs a higher order modulation scheme when the channel is favorable, in order to increase the throughput and conversely, a more robust lower order modulation scheme when the channel exhibits a deep fade in order to improve the mean bit-error rate (BER) performance. Recent developments in AQAM over a narrow-band channel include contributions by Webb and Steele [1], Sampei et al. [2], Torrance et al. [3], and Goldsmith et al. [4]. In these contributions the input SNR was used as the modem mode switching criterion which is not an adequate wide-band channel quality measure, where the transmission burst experiences both power fluctuations and intersymbol interference (ISI). Previous contributions on invoking AQAM over wide-band channels are due, for example, to Morinaga et al. [5] and Lim et al. [6]. In our proposed joint scheme, the equalization process will eliminate most of the ISI. Consequently, the pseudo signal-to-noise ratio (SNR) at the output of the decision-feedback equalizer (DFE), which is defined later, is calculated and used as a criterion to switch the modulation modes.

\section{SYSTEM OVERVIEW}

The joint AQAM and equalization schematic is depicted in Fig. 1. At the receiver, the DFE coefficients are calculated by solving (1) and (2), which will be described later. Additionally, the channel estimates and the DFE coefficients are utilized to compute the pseudo-SNR at the DFE output. The pseudo-SNR is then compared to a set of pseudo-SNR thresholds $f_{n}$, and a modulation scheme for the next transmission burst is selected as shown in Table I.

Paper approved by A. Goldsmith, the Editor for Wireless Communication of the IEEE Communications Society. Manuscript received July 8, 1998; revised November 2, 1998 and February 2, 1999. This work was supported by the European Commission and the EPSRC, Swindon, U.K.

The authors are with the Department of Electrical and Computer Science, University of Southampton, Southampton SO17 1BJ, U.K. (e-mail lh@ecs.soton.ac.uk).

Publisher Item Identifier S 0090-6778(00)02274-1.
TABLE I

Modulation Switching Mechanism Based ON PSEUdo-SNR, $\gamma_{\text {DFE }}$ AND ITS CORRESPONDING SWITCHING THRESHOLDS $f_{n}$, WHERE $\boldsymbol{n}=1,2,3,4$ FOR THE AQAM SYSTEM

\begin{tabular}{c|c}
\hline Modulation Mode & Pseudo SNR Range \\
\hline BPSK & $f_{1} \geq \gamma_{d f e}<f_{2}$ \\
\hline 4QAM & $f_{2} \geq \gamma_{d f e}<f_{3}$ \\
\hline 16QAM & $f_{3} \geq \gamma_{d f e}<f_{4}$ \\
\hline $64 Q A M$ & $\gamma_{d f e} \geq f_{4}$ \\
\hline
\end{tabular}

The symbol-spaced channel impulse response (CIR) used in our investigation is derived from the COST 207 [7] typical urban area (TU) channel model, as shown in Fig. 2, but our subsequent analysis can be generalized for other channel models. Each path is faded independently according to a Rayleigh distribution which assumed a normalized Doppler frequency of $3.3615 \times 10^{-5}$. Variations due to path loss and shadowing are assumed to be eliminated by power control.

For the minimum mean-square-error DFE, the optimum coefficients for the forward filter $C_{m}$ and the backward filter $b_{q}$ can be written as [8]

$$
\begin{gathered}
\sum_{m=0}^{N_{f}-1} C_{m}\left[\sum_{v=0}^{l} h_{v}^{*} h_{v+m-l} \sigma_{S}^{2}+N_{o} \delta_{m-l}\right] \\
=h_{l}^{*} \sigma_{S}^{2}, \quad l=0 \cdots N_{f}-1 \\
b_{q}=\sum_{m=0}^{N_{f}-1} C_{m} h_{m+q}, \quad q=1 \cdots N_{b}
\end{gathered}
$$

where $N_{f}$ and $N_{b}$ represent the number of taps in the forward filter and the backward filter, which were set to 35 and 7, respectively. $\sigma_{S}^{2}$ denotes the variance of the transmitted signal and the superscript $*$ represents complex conjugation. $N_{\circ}$ is the single-sided power spectral density of white noise, $h_{i}$ denotes the $i$ th path of the CIR, and $\delta$ denotes the Dirac delta function. The upper-bound performance of this joint AQAM and equalization scheme was derived by making several assumptions. First, the CIR is constant across the transmission burst, but varies from burst to burst by assuming that the channel is slowly varying. Second, perfect channel compensation and perfect knowledge of the modulation scheme used was assumed at the receiver. In reality, some form of signaling must be used to convey the modulation scheme used to the receiver. Third, the pseudo-SNR at the output of the equalizer is estimated perfectly prior to transmission. This can only be applied in a TDD scenario [9] or when there is a reliable, low-delay feedback path 


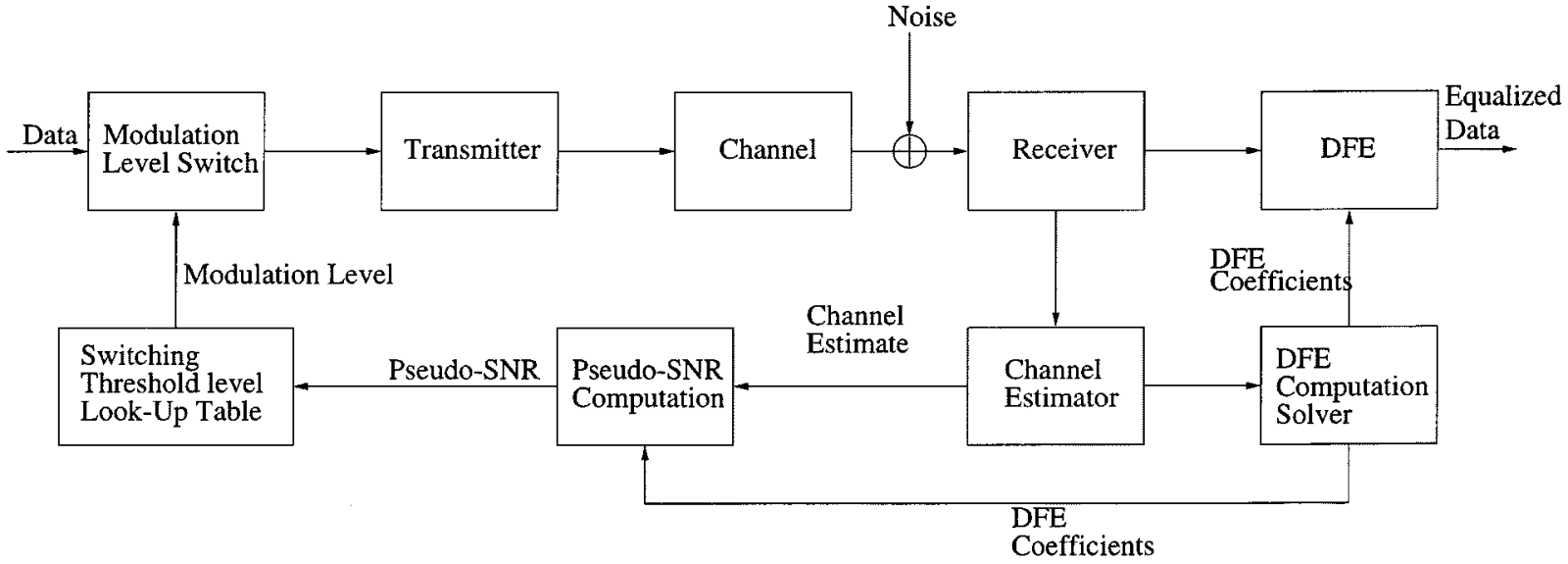

Fig. 1. Schematic of the joint AQAM and equalization scheme.

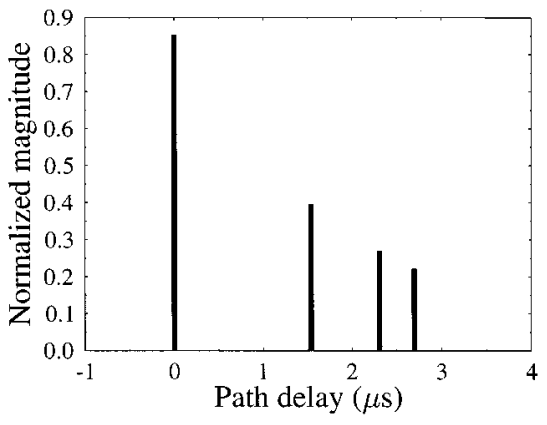

Fig. 2. The impulse response of a COST 207 TU channel.

between the transmitter and the receiver. The error propagation of the DFE will degrade the upper-bound performance. However by using AQAM, the mean BER is lowered which then decreases the probability of error propagation. Therefore, the error propagation is neglected to simplify the analysis.

\section{PSEUDO-SNR OF THE DFE}

We will begin our justification of using the pseudo-SNR as a switching criterion by presenting the equations used to calculate the pseudo-SNR $\gamma_{\mathrm{DFE}}$ as follows [8]:

$$
\begin{aligned}
& \gamma_{\mathrm{DFE}}=\frac{\text { wanted signal power }}{E\left[\left|S_{k} \sum_{m=0}^{N_{f}-1} C_{m} h_{m}\right|^{2}\right]} \\
& \gamma_{\mathrm{DFE}}=\frac{\sum_{q=-\left(N_{f}-1\right)}^{-1} E\left[\left|d_{q} S_{k-q}\right|^{2}\right]+N_{o} \sum_{m=0}^{N_{f}-1}\left|C_{m}\right|^{2}}{\text { residual ISI power + effective noise power }}
\end{aligned}
$$

where $d_{q}=\sum_{m=0}^{N_{f}-1} C_{m} h_{m+q}$ and $S_{k}$ is the transmitted signal at time $k$ which was assumed uncorrelated.

For the pseudo-SNR to be viable as a switching criterion, it has to be able to estimate the mean BER for fixed modulation schemes and consequently for AQAM schemes. However, since it is difficult to characterize the probability density function (pdf) of the residual interference, the residual interference was approximated as additional noise at the output of the DFE.
This was also applied by Monsen [10] who asserted that the interference characteristics do not significantly alter the mean BER performance. To verify this, several experiments were conducted using fixed modulation schemes in a fading multipath environment, where the mean BER performance for each of these schemes, $P_{\mathrm{MODN}_{M R}}^{b}(\gamma)$ was numerically calculated as follows:

$$
P_{\mathrm{MODN}_{M R}}^{b}(\gamma)=\int_{0}^{\infty} P_{\mathrm{MODN}}^{b}\left(\gamma_{\mathrm{DFE}}\right) p\left(\gamma_{\mathrm{DFE}}\right) d \gamma_{\mathrm{DFE}}
$$

where MODN can be for binary phase-shift keying (BPSK), 4-QAM, 16-QAM, or 64-QAM. $p\left(\gamma_{\mathrm{DFE}}\right)$ is the pdf of the instantaneous pseudo-SNR for a given average channel SNR $\gamma$. In order to obtain $p\left(\gamma_{\mathrm{DFE}}\right)$, the instantaneous values of $\gamma_{\mathrm{DFE}}$ were calculated using (3). Subsequently, the discretised pdf was constructed and inserted into (4). The integration was approximated by the trapezoidal rule with the limits set to the lowest and highest values of the instantaneous pseudo-SNR. The notation $P_{\mathrm{MODN}}^{b}$ is the individual theoretical QAM Gaussian BER performance used in our AQAM system [9].

The results shown in Fig. 3 displayed close correspondence between the numerical and simulated performance for each individual modulation scheme. Consequently, the utilization of $\gamma_{\mathrm{DFE}}$ as a modulation switching criterion in the proposed joint scheme is justified.

\section{Numerical AVERAge UpPer-Bound PERFORMANCE}

The numerical method suggested by Torrance [3] is modified for this joint adaptive scheme by using the pseudo-SNR to yield the corresponding numerical mean BER performance:

$$
\begin{aligned}
P_{a}(\gamma)=B^{-1} \cdot[1 & \cdot \int_{f_{1}}^{f_{2}} P_{\mathrm{BPSK}}^{b}\left(\gamma_{\mathrm{DFE}}\right) p\left(\gamma_{\mathrm{DFE}}\right) d \gamma_{\mathrm{DFE}} \\
& +2 \cdot \int_{f_{2}}^{f_{3}} P_{4 \mathrm{QAM}}^{b}\left(\gamma_{\mathrm{DFE}}\right) p\left(\gamma_{\mathrm{DFE}}\right) d \gamma_{\mathrm{DFE}} \\
& +4 \cdot \int_{f_{3}}^{f_{4}} P_{16 \mathrm{QAM}}^{b}\left(\gamma_{\mathrm{DFE}}\right) p\left(\gamma_{\mathrm{DFE}}\right) d \gamma_{\mathrm{DFE}} \\
& \left.+6 \cdot \int_{f_{4}}^{\infty} P_{64 \mathrm{QAM}}^{b}\left(\gamma_{\mathrm{DFE}}\right) p\left(\gamma_{\mathrm{DFE}}\right) d \gamma_{\mathrm{DFE}}\right]
\end{aligned}
$$




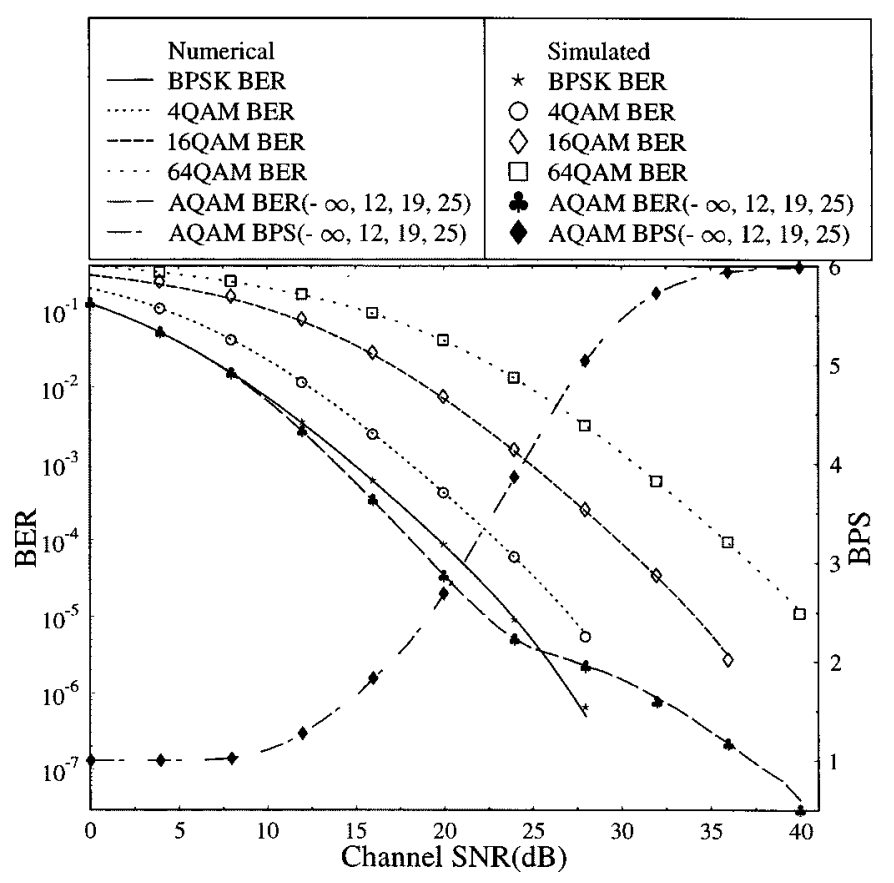

Fig. 3. Numerical and simulated performance of the joint AQAM and DFE, employing BPSK, 4 QAM, 16 QAM, and 64 QAM over the COST 207 TU Rayleigh fading channel of Fig. 2 using the assumptions in Section II. The numerical solutions were calculated using (4) for the fixed modulation schemes and (5) and (6) were used to calculate the numerical solution for the joint AQAM and DFE scheme.

where $B$ is the mean number of bits per symbol (BPS), which can be written as

$$
\begin{aligned}
B= & 1 \cdot \int_{f_{1}}^{f_{2}} p\left(\gamma_{\mathrm{DFE}}\right) d \gamma_{\mathrm{DFE}}+2 \cdot \int_{f_{2}}^{f_{3}} p\left(\gamma_{\mathrm{DFE}}\right) d \gamma_{\mathrm{DFE}} \\
& +4 \cdot \int_{f_{3}}^{f_{4}} p\left(\gamma_{\mathrm{DFE}}\right) d \gamma_{\mathrm{DFE}}+6 \cdot \int_{f_{4}}^{\infty} p\left(\gamma_{\mathrm{DFE}}\right) d \gamma_{\mathrm{DFE}} .
\end{aligned}
$$

The joint AQAM and equalization numerical upper-bound performance over the COST 207 TU Rayleigh fading channel is calculated similarly to the fixed modulation mean BER numerical performance discussed in Section III. The threshold levels were set as follows: $f_{1}=-\infty \mathrm{dB}, f_{2}=12 \mathrm{~dB}, f_{3}=19$ $\mathrm{dB}$, and $f_{4}=25 \mathrm{~dB}$. These levels were set such that a BER of approximately $1 \%$ was maintained according to the fixed modulation performance of Fig. 3. The results are shown in Fig. 3 where there was a good correspondence between the numerical and simulated performance of the joint AQAM and equalization scheme.
In the AQAM scheme, for channel SNR's lower than $25 \mathrm{~dB}$, the mean BER and BPS performances are better or equal to the performance using BPSK. At higher channel SNR's, the mean BER and BPS performance begins to converge to the 64-QAM performance due to the low probability of encountering a low instantaneous pseudo-SNR. Since the mean BPS is higher, the adaptive mean BER performance can even be lower when compared to the BPSK performance due to the averaging over a higher number of bits.

\section{CONCLUSIONS}

The application of AQAM to a wide-band channel environment was extended by jointly exploiting the benefits of AQAM and equalization. A new criterion, the pseudo-SNR at the output of the DFE was introduced to switch the modulation modes and consequently, a numerical upper-bound performance characterized by (5) and (6) was presented.

\section{ACKNOWLEDGMENT}

The authors would like to thank the various anonymous reviewers for their invaluable comments and suggestions.

\section{REFERENCES}

[1] W. T. Webb and R. Steele, "Variable rate qam for mobile radio," IEEE Trans. Commun., vol. 43, pp. 2223-2230, July 1995.

[2] S. Sampei, S. Komaki, and N. Morinaga, "Adaptive modulation/TDMA scheme for large capacity personal multimedia communications systems," IEICE Trans. Commun., vol. E77-B, pp. 1096-1103, Sept. 1994.

[3] J. M. Torrance and L. Hanzo, "On the upper bound performance of adaptive QAM in a slow Rayleigh fading," Electron. Lett., vol. 32, pp. 169-171, Apr. 1996.

[4] A. Goldsmith and S. G. Chua, "Variable rate variable power MQAM for fading channels," IEEE Trans. Commun., vol. 45, pp. 1218-1230, Oct. 1997.

[5] S. Sampei, N. Morinaga, and Y. Kamio, "Adaptive modulation/TDMA with a BDDFE for $2 \mathrm{Mbit} / \mathrm{s}$ multi-media wireless communication systems," in Proc. Vehicular Technology Conf., vol. 1, July 1995, pp. 311-315.

[6] C. H. Lim and J. K. Jeong, "Adaptive modulation using the multipath fading compensation," IEE Electron. Lett., vol. 34, pp. 940-942, 1998.

[7] "COST 207: Digital land mobile radio communications-Final report," Office for Official Publications of the European Communities, Luxembourg, Final Rep., 1989.

[8] J. C. Cheung, "Adaptive equalisers for wideband TDMA mobile radio," Ph.D. dissertation, University of Southampton, Southampton, U.K., 1991.

[9] L. Hanzo, W. T. Webb, and T. Keller, Single- and Multicarrier Quadrature Amplitude Modulation. New York: IEEE Press/Wiley, 1994.

[10] P. Monsen, "Theoretical and measured performance of a DFE modem on a fading multipath channel," IEEE Trans. Commun., vol. COM-25, pp. 1144-1153, Oct. 1977. 